\title{
Controllable synthesis and spontaneous phase transition of photonic coordination polymer to produce a strong second-harmonic generation response
}

\author{
Jun Zhang ${ }_{4^{*}}^{1 \dagger^{*}}$, Maierhaba Abudoureheman ${ }^{2,4 \dagger}$, Xiaofan $\mathrm{Ma}^{3}$, Weili Kong ${ }^{1}$, Xiaopeng Xuan ${ }^{3^{*}}$ and \\ Shilie $\operatorname{Pan}^{4 *}$
}

\begin{abstract}
Herein, a novel photonic coordination polymer material was constructed by aggregation-induced emission luminogen (AIEgen) containing a tripyridyl moiety used as the linking ligand. It displayed a spontaneous direct centrosymmetric to noncentrosymmetric phase transition in a single crystal. The two crystals, before and after the phase transition, were both controllably synthesized and characterized by single-crystal $\mathrm{X}$-ray diffraction. After being exposed to air, the centrosymmetric metastable phase (1-a) transitioned to a new stable phase with a noncentrosymmetric structure (1$\boldsymbol{\beta})$. Interestingly, the $1-\boldsymbol{\beta}$ structure exhibited a strong phasematching second-harmonic generation (SHG) response, about 4.5 times higher than that of $\mathrm{KH}_{2} \mathrm{PO}_{4}$ (KDP). In order to better understand the relationship between the structure and the nonlinear optical properties, the dipole moments were calculated and discussed. Remarkably, the noncentrosymmetric phase with high thermal stability for $1-\beta$ retained and improved the initial photoluminescent properties of the AIEgen ligand after the structural phase transition from 1- $\alpha$, and simultaneously produced the excellent SHG property, which are beneficial for the design and construction of excellent optical materials.
\end{abstract}

Keywords: second-harmonic generation, controllable synthesis, phase transition, crystal structure, single-crystal X-ray diffraction

\section{INTRODUCTION}

Nonlinear optical (NLO) materials play an important role in modern laser science and technology. Intensive studies have been focused on exploring NLO materials with enhanced second harmonic generation (SHG) [1-4]. Enhancing the SHG signals through phase transitions can be associated with temperature or pressure changes [5-7]. Over the past few decades, a number of famous NLO crystals have been obtained such as $\mathrm{CsBsOs}(\mathrm{CBO})[8]$, $\mathrm{LiB}_{3} \mathrm{O}_{5}$ (LBO) [9], $\mathrm{CsLiB}_{6} \mathrm{O}_{10}$ (CLBO) [10], $\mathrm{K}_{3} \mathrm{~B}_{6} \mathrm{O}_{10} \mathrm{Cl}$ (KBOC) [2], $\mathrm{Ba}_{3} \mathrm{~B}_{6} \mathrm{O}_{11} \mathrm{~F}_{2}$ [11], and $\mathrm{Cs}_{2} \mathrm{~B}_{4} \mathrm{SiO}_{9}$ [12]. Considering their superiorities, borates have been widely studied, and even now promising new borate compounds are being discovered [13-16], even though their synthetic conditions are severe and their structures are difficult to tune $[17,18]$. Recently, controllable NLO switches, with reversible SHG conversion between different states by external stimulus [19], have become an exciting new branch of NLO materials. Remarkably, developing a promising strategy for solid-state NLO switches relies on a structural phase transition to realign the noncentrosymmetric NLO moieties and alter their SHG signals, where those with a transition from a centrosymmetric structure to a noncentrosymmetric structure achieve a sharp NLO response change. Understanding such an exceptional phase transition and utilizing it to achieve a new type of NLO switch that activates SHG by heating are meaningful. In contrast to the inorganic NLO materials, the organic-inorganic hybrid materials with NLO properties are seldom reported

\footnotetext{
${ }^{1}$ School of Materials and Chemical Engineering, Anhui Jianzhu University, Hefei 230601, China

${ }^{2}$ College of Chemistry and Chemical Engineering, Key Laboratory of Coal Clean Conversion \& Chemical Engineering Process, College of Chemistry and Chemical Engineering, Xinjiang University, Urumqi 830046, China

${ }^{3}$ School of Chemistry and Chemical Engineering, Key Laboratory of Green Chemical Media and Reactions, Ministry of Education, Collaborative Innovation Center of Henan Province for Green Manufacturing of Fine Chemicals, Henan Normal University, Xinxiang 453007, China

${ }^{4}$ CAS Key Laboratory of Functional Materials and Devices for Special Environments, Xinjiang Technical Institute of Physics \& Chemistry, CAS; Xinjiang Key Laboratory of Electronic Information Materials and Devices, Urumqi 830011, China

† These two authors contributed equally to this work.

* Corresponding authors (emails: zhangjun@ahjzu.edu.cn (Zhang J); xpxuan@henannu.edu.cn (Xuan X); slpan@ms.xjb.ac.cn (Pan S))
} 
$[20,21]$. Also, their further applications are limited due to their weak thermal stability.

Alternatively, aggregation-induced emission (AIE) luminogens (AIEgens) have potential applications in optoelectronic devices, fluorescent sensors, biological imaging, and other fields [22]. Their radiative channels are opened by restrictions of vibration (RIR) or other mechanisms when aggregation occurs due to the addition of incompatible solvents [23]. The solubility of AIEgens affects their photophysical properties. Moreover, organic molecules are detrimental to AIEgens' applications due to their single organic skeleton, low melting point, and low thermal stability. The RIR of AIEgens is permanently inhibited in the crystal lattice from coordination with various metals; thus solid-state coordination polymers (CPs) constructed by AIE ligands could maintain the photophysical properties of the AIEgens' aggregated state, display novel long-distance order structures [24], and even produce special properties that are different from AIEgens.

Moreover, $\mathrm{CPs}$ can possess various structures through the alteration of organic linkers and the coordination styles of metal atoms, allowing for the ability to conveniently tune the properties of CPs. For example, the Vtype $p$-dipyridine derivatives $[20,25]$ can be coordinated with zinc ions to form noncentrosymmetric complexes through structural distortion. Altering organic linkers and metal atoms is a good approach to controllably synthesize and tune the structures of CPs using AIEgen ligands for maintaining the original properties of organics; however, they are still challenging for current researchers.

Herein, we rationally designed and synthesized a $D$ (donor)-A (acceptor) type organic molecule 4'-(4-(di- $p$ tolylmethyl)phenyl)-4,2':6',4"-terpyridine (denoted as $\gamma$ dptpt) possessing AIE properties and a $\mathrm{V}$-shaped geometry, and coordinated it with $\mathrm{Zn}(\mathrm{II})$ ions, often adopted to distort the tetrahedral geometry, to obtain a novel one-dimensional (1D) CP with two phases (abbreviated as $\mathbf{1 - \alpha}$ and $\mathbf{1 - \beta}$ ). Both can be controllably synthesized through changes in temperature or dryness of the solvent. The breakaway of guest solvents results in a direct phase transition from 1- $\boldsymbol{\alpha}$ to $\mathbf{1 - \beta}$. Surprisingly, $\mathbf{1 - \beta}$ inherits and improves the excellent properties of the AIEgen as the linked ligand and even produces superior phase-matching SHG signals compared with the centrosymmetrical stacking of $\boldsymbol{\gamma}$-dptpt and 1-a. Additionally, $\mathbf{1 - \beta}$ is stable up to about $455^{\circ} \mathrm{C}$, indicating it has better thermal stability than common organics and comparable to inorganics.

\section{EXPERIMENTAL SECTION}

\section{Controllable synthesis of the $1-\alpha$ and $1-\beta$ phases}

To a solution of $\gamma$-dptpt $(0.0504 \mathrm{~g}, 0.10 \mathrm{mmol})$ in $6 \mathrm{~mL}$ $\mathrm{CHCl}_{3}$ and $1 \mathrm{~mL} \mathrm{CH} \mathrm{CH}_{3} \mathrm{OH}$ were slowly added to $3 \mathrm{~mL}$ $\mathrm{CH}_{3} \mathrm{OH}$ in a test tube. Then, they were carefully layered in a $10 \mathrm{~mL}$ methanolic solution of $\mathrm{ZnCl}_{2}(0.0136 \mathrm{~g}$, $0.10 \mathrm{mmol})$. The tube was sealed and kept at $10^{\circ} \mathrm{C}$ for several days, and yellow block-like crystals (1-a) were obtained with a yield of about $76.2 \%$.

According to the same procedure but with altered temperature, orange crystals $(0.052 \mathrm{~g})$ of compound $\mathbf{1 - \beta}$ were successfully prepared at $30^{\circ} \mathrm{C}$ with a $77.4 \%$ yield. Elemental analysis (EA): Calcd. H: $4.79 \%$; C: $64.25 \%$. Exp.: H: 4.88\%; C: $64.10 \%$.

\section{SHG measurements}

The fundamental wavelength $1064 \mathrm{~nm}$ was generated by a Q-switched Nd:YAG laser. 1- $\boldsymbol{\beta}$ was ground and sieved into different distinct particle size ranges: $<20,20-38$, 3855, 55-88, 88-105, 105-150, and 150-200 mm. Microcrystalline $\mathrm{KH}_{2} \mathrm{PO}_{4}(\mathrm{KDP})$ was also ground and sieved into the same particle size ranges and used as reference.

\section{RESULTS AND DISCUSSION}

\section{Crystallographic data}

As shown in Tables S1-6 and Fig. S1, compound 1-a crystallizes in a monoclinic system $C 2 / c$ space group. Its asymmetric unit contains one $\mathrm{Zn}(\mathrm{II})$ atom, one $\boldsymbol{\gamma}$-dpdpt ligand, two $\mathrm{Cl}$ atoms, and two guest water molecules. Different from 1- $\boldsymbol{\alpha}, \mathbf{1 - \beta}$ crystallizes in an orthorhombic system $P n a 2_{1}$ space group. The Flack parameter of 0.005 (7) confirmed the absolute configuration [26]. In the asymmetric unit of $\mathbf{1} \boldsymbol{\beta} \boldsymbol{\beta}$, the two guest water molecules of $\mathbf{1 - \alpha}$ were replaced by a guest methanol molecule, and the structure was transformed from centrosymmetry to noncentrosymmetry due to the rotation of the pyridine ring. In these two compounds, the $\mathrm{Zn}$ (II) atom adopts a tetrahedron geometry to coordinate with two $\mathrm{Cl}^{-}$anions and two $\mathrm{N}$ atoms from two different $\boldsymbol{\gamma}$-dptpt ligands to propagate a 1D zig-zag chain (Fig. 1). With respect to the $\mathrm{Zn}(\mathrm{II})$ chain, the $\boldsymbol{\gamma}$-dptpt ligands array exists bilaterally for $\mathbf{1 - \alpha}$ or ipsolaterally for $\mathbf{1 - \beta}$, just like the states of a butterfly's opening/closing wings. The $1 \mathrm{D}$ band propagates the 3D network by an $\mathrm{ABAB}$ stacking model along the $\boldsymbol{a}$-axes of $\mathbf{1 - \alpha}$ and $\mathbf{1 - \beta}$ (Fig. S2).

By comparing the geometries of compounds 1- $\boldsymbol{\alpha}$ and $\mathbf{1 -}$ $\beta$, we found that the opening-closing ligands can be attributed to the significant distortion of aromatic benzene 


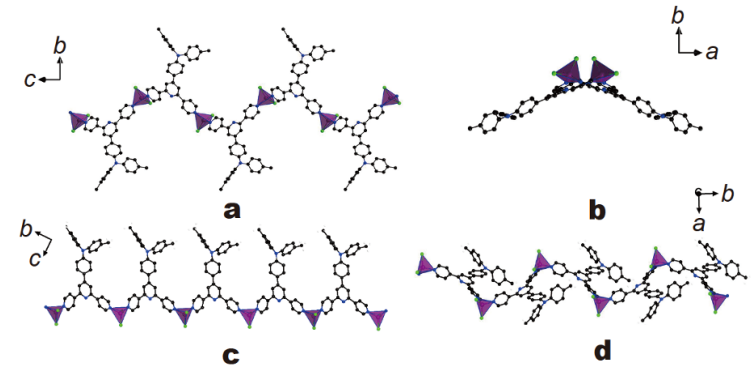

Figure 1 1D chain structures of $\mathbf{1 - \alpha}(\mathrm{a}, \mathrm{b})$ and $\mathbf{1 - \beta}(\mathrm{c}, \mathrm{d})$. The H-atoms and the solvent molecules are omitted for clarity.

and pyridine rings, and their rotations are shown in Fig. S3a. In 1-a, the dihedral angles between the three coplanar pyridine rings $\mathrm{P} 1, \mathrm{P} 2$, and $\mathrm{P} 3$ were $2.01^{\circ}, 6.70^{\circ}$, and $6.70^{\circ}$, respectively. However, they were $20.44^{\circ}, 25.51^{\circ}$, and $42.60^{\circ}$ in $\mathbf{1}-\boldsymbol{\beta}$, and three pyridine rings were also strongly distorted. In addition, the dihedral angles between the pyridine $(\mathrm{P} 1)$ and benzene rings $(\mathrm{Pa})$ were $37.16^{\circ}$ for $\mathbf{1}-\boldsymbol{\alpha}$ and $20.65^{\circ}$ for $\mathbf{1}-\boldsymbol{\beta}$. Furthermore, the bond length of the phases also changed slightly. The $\mathrm{Zn}-\mathrm{Cl} 1$ bond length changed from 2.230(2) $\AA$ in $\mathbf{1 - \alpha}$ to $2.211(2) \AA$ in $\mathbf{1}-\boldsymbol{\beta}, \mathrm{Zn}-\mathrm{Cl} 2$ from $2.240(2) \AA$ in $\mathbf{1}-\boldsymbol{\alpha}$ to 2.226(1) $\AA$ in $\mathbf{1 - \beta}$, and $\mathrm{Zn}-\mathrm{N}$ bond length changed from $2.071(4) \AA$ in $\mathbf{1}-\boldsymbol{\alpha}$ to $2.048(2) \AA$ in $\mathbf{1}-\boldsymbol{\beta}$. A more compact coordination structure in $\mathbf{1}-\boldsymbol{\beta}$ is one of the factors affecting the crystal transformation [27].

\section{Powder X-ray diffraction (PXRD) analysis and density functional theory (DFT) calculations}

As depicted in Fig. S4, 1- $\boldsymbol{\beta}$ phase is stable both in the original liquor and in air. Although 1- $\boldsymbol{\alpha}$ is also stable in the original liquor, it completely loses luster within ca. 1 min after being exposed to air. The PXRD further confirms that the 1-a framework successfully transformed into the isomeric 1- $\boldsymbol{\beta}$. The crystal transformation of $\mathbf{1 - \alpha}$ was probably generated from the breakaway of guest water in the ambient conditions since its structures were induced and stabilized in the hydrous conditions. When the completely anhydrous solvents were applied to synthesize 1- $\boldsymbol{\alpha}$ under the same conditions, $\mathbf{1 -} \boldsymbol{\beta}$ rather than 1$\boldsymbol{\alpha}$ was obtained. When the temperature was increased to $30^{\circ} \mathrm{C}$ for the synthesis of $\mathbf{1 - \alpha}, \mathbf{1}-\boldsymbol{\beta}$ was obtained instead. Obviously, the guest water molecules stabilize the structure of 1-a, and the breakaway of water results in the crystal transformation from 1- $\boldsymbol{\alpha}$ to $\mathbf{1 - \beta}$ via a phase transition, which is different from the mechanical stress mechanism [28]. Furthermore, according to the aforementioned structural characterization, the variation of configuration from $\mathbf{1}-\boldsymbol{\alpha}$ to $\mathbf{1}-\boldsymbol{\beta}$ also demonstrates $180^{\circ}$ rolling-over molecular motions even under the restrictions of the solid-state lattice.

DFT calculations based on the single-crystal structures were performed on the model structures of $\mathbf{1 - \alpha}$ and $\mathbf{1 - \beta}$ to obtain mechanistic insight into their stable properties. Calculations indicate that the total energy for $\mathbf{1 - \alpha}$ is lower than that for $\mathbf{1 - \beta}$, revealing that $\mathbf{1 - \alpha}$ is thermodynamically unfavorable compared with 1- $\boldsymbol{\beta}$. The absorption energy gap from $2.86 \mathrm{eV}$ for $\mathbf{1}-\boldsymbol{\alpha}$ to $2.94 \mathrm{eV}$ for $\mathbf{1 - \beta}$ (Fig. S3b) and the more compact coordination structure of $\mathbf{1 - \beta}$ are also responsible for the structural change between $\mathbf{1 - \alpha}$ and $\mathbf{1 - \beta}$.

\section{Thermogravimetric (TG) and differential scanning calorimetry (DSC)}

Since $\mathbf{1 - \alpha}$ can transform into $\mathbf{1 - \beta}$ in the air at ambient temperature, TG and DSC determination were only performed on 1- $\boldsymbol{\beta}$. As shown in Fig. 2, with increased temperature the TG curve slowly decreased and lost a total of $2.3 \%$ (calculated $4.8 \%$ ) at $455^{\circ} \mathrm{C}$, which can be attributed to the loss of guest methanol. Subsequently, a sharp drop appeared due to thermal decomposition. These reveal that the structure of $\mathbf{1} \boldsymbol{\beta}$ can be retained below $455^{\circ} \mathrm{C}$ in accordance with the DSC. The thermal stability of $\mathbf{1 - \beta}$ was significantly higher than that of most CPs [29-31], and therefore it can be used to achieve practical needs [32].

\section{AIE effect of $\boldsymbol{\gamma}$-dptpt}

Intermolecular stacking and large conjugate systems endow the AIE properties of the $\boldsymbol{\gamma}$-dptpt ligand. As shown in Fig. 3 and Fig. S5, $\boldsymbol{\gamma}$-dptpt shows a strong yellow fluorescence in dimethyl formamide (DMF). With an increased water volume fraction, the fluorescence of $\boldsymbol{\gamma}$-dptpt decreased significantly. When the ratio of water

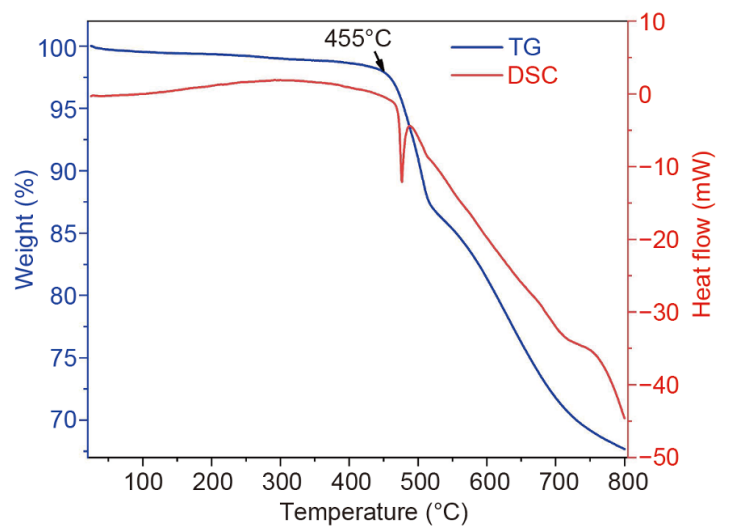

Figure 2 TG and DSC plots of $\mathbf{1 - \beta}$ from room temperature to $800^{\circ} \mathrm{C}$ under $\mathrm{N}_{2}$ flow. 


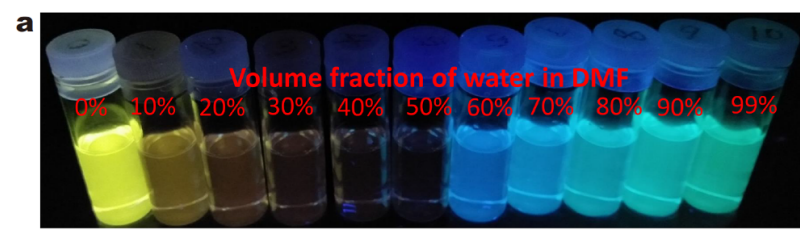

b

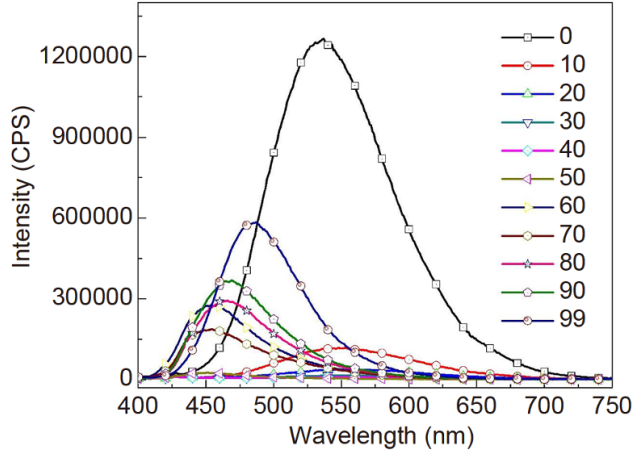

c

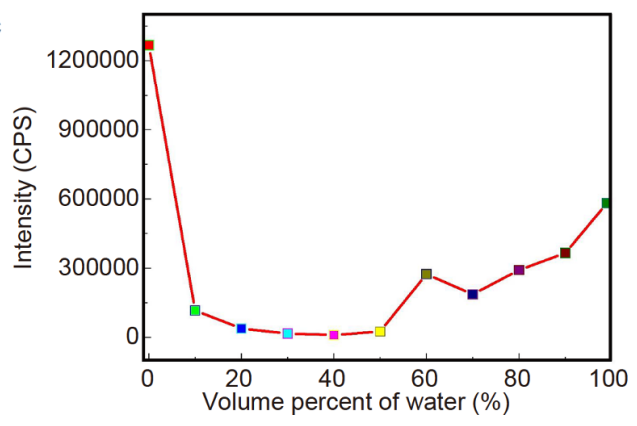

Figure 3 (a) Photographs of $\boldsymbol{\gamma}$-dptpt in DMF/water mixtures with 0 , $10 \%, 20 \%, 30 \%, 40 \%, 50 \%, 60 \%, 70 \%, 80 \%, 90 \%$ and $100 \%$ water contents taken in the presence of $365 \mathrm{~nm}$ UV irradiation from a hand-held UV lamp. (b) Photoluminescence (PL) spectra $\left(\lambda_{\mathrm{ex}}=360 \mathrm{~nm}\right)$ of $\boldsymbol{\gamma}$-dptpt in DMF/water mixtures with different water fractions $\left(f_{\mathrm{w}}\right)$. (c) PL intensity at $\lambda_{\mathrm{em}}=550 \mathrm{~nm}$ of $\boldsymbol{\gamma}$-dptpt aggregates formed in DMF/water mixtures with different $f_{\mathrm{w}}$.

to DMF was $1: 1$, the fluorescence intensity began to recover. Then, with the increase of water, the fluorescence peaks underwent an obvious blue-shift, and the intensity strongly increased. The nanoparticle size experiments showed that the size of the nanoparticles changed obviously when the ratio of $\mathrm{DMF} /$ water was $1: 1$, which corresponded to the change of fluorescence intensity. The above results show that the self-aggregation of $\boldsymbol{\gamma}$-dptpt occurs with increasing water ratio, which results in a blue-shift of the fluorescence emission peak and the enhancement of fluorescence. This is a typical phenomenon of the AIE property.

\section{Solid-state optical diffuse reflection and fluorescence} emission measurement

The solid-state optical diffuse reflection and fluorescence spectra of $\boldsymbol{\gamma}$-dpdpt and $\mathbf{1}-\boldsymbol{\beta}$ were measured at room temperature as shown in Fig. $4 \mathrm{a}$ and b. The ultravioletvisible (UV-Vis) absorption and emission spectra of $\mathbf{1 - \beta}$ indicate that the as-prepared compound has nearly no shift in the UV-Vis maximum absorption compared with that of $\boldsymbol{\gamma}$-dptpt, and the intense emission band centered at $530 \mathrm{~nm}$ was attributed to the $\pi^{*} \rightarrow \mathrm{n}$ or $\pi^{*} \rightarrow \pi$ transitions [33]. The fluorescence peaks underwent an obvious red-shift, which may be assigned to a ligand to ligand charge transfer (LLCT) transition [34,35]. To further investigate the optical properties, the quantum yields, and fluorescence lifetimes of the ligand and 1- $\beta$ were studied. The solid-state photophysical parameters of $\boldsymbol{\gamma}$-dptpt and $\mathbf{1}-\boldsymbol{\beta}$ are presented in Fig. $4 \mathrm{c}$ and d. $\mathbf{1}-\boldsymbol{\beta}$ phase has a long $\tau$ of $14.6 \mathrm{~ns}$, about 8 times that of $\gamma$-dptpt (1.9 ns). The quantum yield increases slightly from $10.0 \%$ for $\boldsymbol{\gamma}$-dptpt to $11.2 \%$ for $\mathbf{1 - \beta}$. Once the ligand $\boldsymbol{\gamma}$-dptpt coordinates to $\mathrm{Zn}(\mathrm{II})$, the ligation enhances the rigidity of the ligands and reduces the nonradiative energy loss; thus the fluorescence lifetime of the 1- $\boldsymbol{\beta}$ phase is much stronger than that of the free ligand. These reveal that the 1- $\boldsymbol{\beta}$ phase inherits and even improves the excellent fluorescent properties of $\boldsymbol{\gamma}$-dptpt by coordination.

\section{SHG properties and structure-property relationships}

The reported 1- $\boldsymbol{\beta}$ phase crystallizes in the polar space group $P n a 2_{1}$; thus the SHG effects were considered and measured using the Kurtz-Perry method [36]. The obtained results indicate that the phase-matching SHG response of $1-\boldsymbol{\beta}$ is about 4.5 times that of $\mathrm{KDP}$ and $\sim 0.8$ times that of $\beta-\mathrm{BaB}_{2} \mathrm{O}_{4}$ (BBO) (Fig. 5) [11,12]. To further illustrate the SHG intensity, as well as the structure-SHG property relationship, the magnitudes of the dipole moments for the SHG active structural units were calculated by a bond valence method [37]. It is worth noting that the $\mathrm{ZnCl}_{2} \mathrm{~N}_{2}$ tetrahedra with $\mathrm{d}^{10} \mathrm{Zn}^{2+}$ cations in the $\mathbf{1}-\boldsymbol{\beta}$ phase may be NLO active groups; thus the dipole moments of the $\mathrm{ZnCl}_{2} \mathrm{~N}_{2}$ tetrahedron were calculated by the famous Debye equation, $\mu=n e R$ (where $\mu$ is the net dipole moment in Debye $\left(10^{-18}\right.$ esu $\left.\mathrm{cm}\right), n$ the total number of electrons, $e$ the charge on an electron, $-4.8 \times 10^{-10} \mathrm{esu}$, and $R$ the difference, in $\mathrm{cm}$, between the "centroids" of positive and negative charge) [37]. Bond-valence theory ( $S_{i}=\exp \left[\left(R_{\mathrm{o}}-R_{\mathrm{i}} / \mathrm{B}\right]\right.$, where $R_{\mathrm{o}}$ is an empirical constant, $R_{i}$ is the length of the bond " $i$ " in $\AA$, and $\mathrm{B}=0.37$ ) was used to estimate the distribution of electrons [38]. The calculation on $\mathrm{MoO}_{3} \mathrm{~F}_{3}$ [37], $\mathrm{MoO}_{4}$ [37], $\mathrm{TeO}_{x}$ [39], and $\mathrm{ZnO}_{4}$ [40] polyhedra confirmed the validity of this method. It should be emphasized here that the cooperative action of the polyhedral dipoles was evaluated using the complete crystal symmetry, including both point and translation 

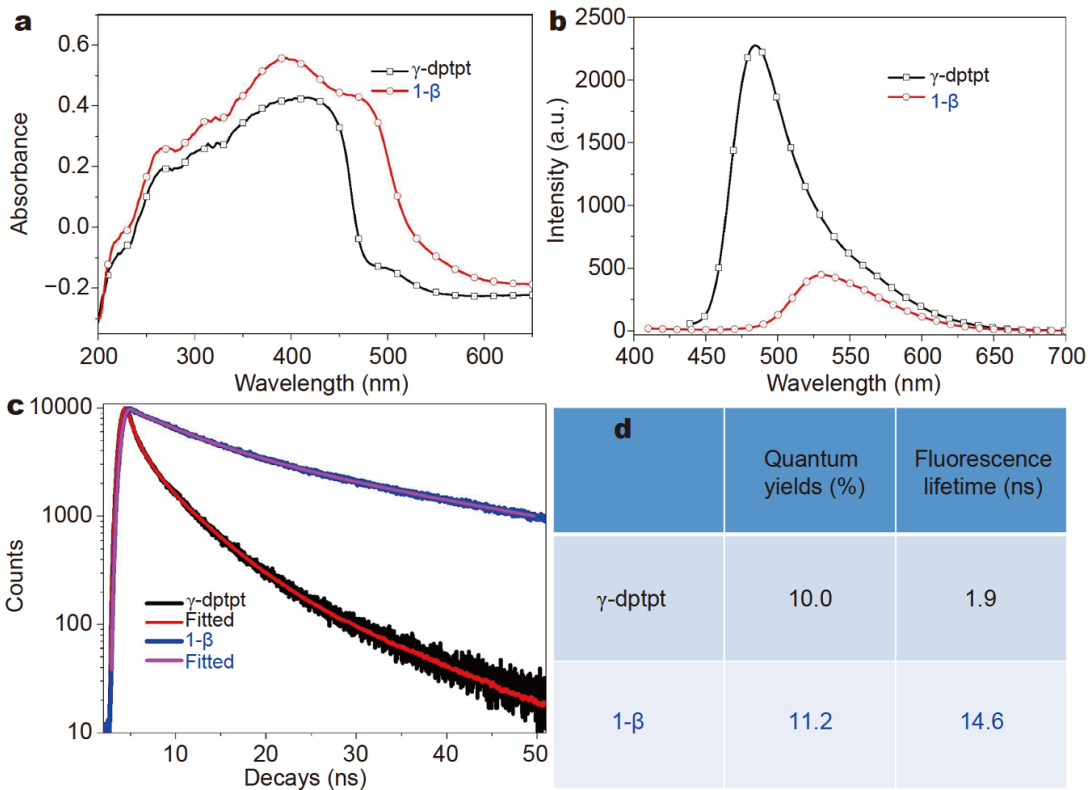

Figure 4 (a) The solid-state diffuse reflectance UV-Vis spectra for the ligand $\boldsymbol{\gamma}$-dptpt and $\mathbf{1 - \beta}$; (b) the solid-state emission spectra for the $\boldsymbol{\gamma}$-dptpt ligand (excited at $419 \mathrm{~nm}$ ) and $\mathbf{1 - \beta}$ (excited at $393 \mathrm{~nm}$ ); (c) fluorescence lifetimes of the $\boldsymbol{\gamma}$-dptpt ligand and $\mathbf{1 - \beta}$ derived from a least-square fit using a single exponential function; (d) fluorescence quantum yields and lifetimes of the $\boldsymbol{\gamma}$-dptpt ligand and $\mathbf{1}-\boldsymbol{\beta}$.

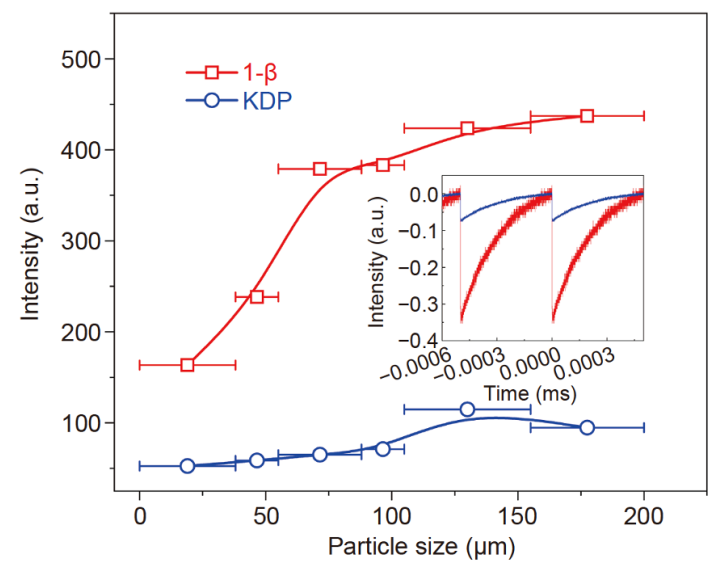

Figure $\mathbf{5}$ SHG intensities of the $\mathbf{1 - \beta}$ phase with commercial KDP as reference: oscilloscope traces from the same particle size $(150-200 \mu \mathrm{m})$ of KDP and 1- $\beta$ powders. operations. Both the contribution from a single $\mathrm{ZnCl}_{2} \mathrm{~N}_{2}$ tetrahedron of the asymmestric unit and the total polarization of the whole unit cell $(Z=4)$ were considered. The detailed calculation results are given in Table 1 . Calculated results show that the differences in the dipole moments of the $\mathrm{ZnCl}_{2} \mathrm{~N}_{2}$ tetrahedron from the symmetric codes were $2.690,2.695,2.694$, and $2.689 \mathrm{D}$, respectively. The magnitudes of the dipole moments along the $a b$ plane nearly canceled, and their vector sum was well enhanced along the $c$-axis.

\section{CONCLUSIONS}

In summary, Zn-CPs with a dipyridyl moiety afforded the centrosymmetric structure of the 1- $\boldsymbol{\alpha}$ phase, while the $\mathbf{1 - \beta}$ phase had a noncentrosymmetric structure. The singlecrystal and PXRD analyses revealed that the centrosymmetric 1-a spontaneously transformed to non-

Table 1 Detailed contribution of the $\mathrm{ZnCl}_{2} \mathrm{~N}_{2}$ tetrahedra from different symmetry codes and total polarization of the whole unit cell $(Z=4)$

\begin{tabular}{ccccc}
\hline Compound $1-\beta$ & \multicolumn{3}{c}{ Dipole moment } \\
\cline { 2 - 5 } Symmetric code & $x$ & $y$ & $z$ & Magnitude (Debye) \\
\hline$x, 1+y, z$ & 1.733 & -1.371 & 1.533 & 2.690 \\
$1 / 2-x, 1 / 2+y,-1 / 2+z$ & -1.738 & -1.372 & 1.536 & 2.695 \\
$1-x,-y,-1 / 2+z$ & -1.738 & 1.370 & 1.536 & 2.694 \\
$1 / 2+x, 1 / 2-y, \mathbf{z}$ & 1.733 & 1.369 & 1.534 & 2.689 \\
$Z=4$ & & & \\
Total polarization & -0.011 & -0.004 & 6.140 & 6.140 \\
\hline
\end{tabular}


centrosymmetric 1- $\boldsymbol{\beta}$ to produce an excellent SHG response. The changes in stabilities and optical properties between 1- $\boldsymbol{\alpha}$ and 1- $\boldsymbol{\beta}$ were correlated with the crystalline structural changes during the water volatilization of 1-a. For the first time, a water-induced metastable to stable crystal transition with a concomitant SHG property change is reported. The high thermal stability of $\mathbf{1 - \beta}$ exhibits a phase-matching SHG response about 4.5 times that of KDP, and the structure-SHG property relationship is discussed. This work demonstrates that the dynamic alteration of stable crystalline phases is a promising strategy for designing universal solvent-responsive functional materials, and explains the intrinsic structural changes to the phase transition.

\section{Received 17 January 2020; accepted 25 February 2020;} published online 8 April 2020

1 Ok KM. Toward the rational design of novel noncentrosymmetric materials: Factors influencing the framework structures. Acc Chem Res, 2016, 49: 2774-2785

2 Wu H, Pan S, Poeppelmeier KR, et al. $\mathrm{K}_{3} \mathrm{~B}_{6} \mathrm{O}_{10} \mathrm{Cl}$ : A new structure analogous to perovskite with a large second harmonic generation response and deep UV absorption edge. J Am Chem Soc, 2011, 133: $7786-7790$

3 Miao Z, Yang Y, Wei Z, et al. A new barium-containing alkali metal silicate fluoride $\mathrm{NaBa}_{3} \mathrm{Si}_{2} \mathrm{O}_{7} \mathrm{~F}$ with deep-UV optical property. Sci China Mater, 2019, 62: 1454-1462

4 Huang J, Guo S, Zhang Z, et al. Designing excellent mid-infrared nonlinear optical materials with fluorooxo-functional group of $\mathrm{d}^{0}$ transition metal oxyfluorides. Sci China Mater, 2019, 62: 1798-1806

5 Kwon OP, Kwon SJ, Jazbinsek M, et al. New organic nonlinear optical polyene crystals and their unusual phase transitions. Adv Funct Mater, 2007, 17: 1750-1756

6 Akiyoshi R, Hirota Y, Kosumi D, et al. Ferroelectric metallomesogens composed of achiral spin crossover molecules. Chem Sci, 2019, 10: 5843-5848

7 Hostettler M, Schwarzenbach D, Helbing J, et al. Structure and SHG of the high pressure phase IV of $\mathrm{HgBr}_{2}$. Solid State Commun, 2004, 129: 359-363

8 Wu Y, Sasaki T, Nakai S, et al. $\mathrm{CsB}_{3} \mathrm{O}_{5}$ : A new nonlinear optical crystal. Appl Phys Lett, 1993, 62: 2614-2615

9 Chen $\mathrm{C}, \mathrm{Wu} \mathrm{Y}$, Jiang $\mathrm{A}$, et al. New nonlinear-optical crystal: $\mathrm{LiB}_{3} \mathrm{O}_{5}$. J Opt Soc Am B, 1989, 6: 616-621

10 Mori Y, Kuroda I, Nakajima S, et al. New nonlinear optical crystal: Cesium lithium borate. Appl Phys Lett, 1995, 67: 1818-1820

$11 \mathrm{Yu} \mathrm{H}, \mathrm{Wu} \mathrm{H}, \mathrm{Pan} \mathrm{S}$, et al. A novel deep UV nonlinear optical crystal $\mathrm{Ba}_{3} \mathrm{~B}_{6} \mathrm{O}_{11} \mathrm{~F}_{2}$, with a new fundamental building block, $\mathrm{B}_{6} \mathrm{O}_{14}$ group. J Mater Chem, 2012, 22: 9665-9670

$12 \mathrm{Wu} \mathrm{H}, \mathrm{Yu} \mathrm{H}, \mathrm{Pan} \mathrm{S}$, et al. $\mathrm{Cs}_{2} \mathrm{~B}_{4} \mathrm{SiO}_{9}$ : A deep-ultraviolet nonlinear optical crystal. Angew Chem Int Ed, 2013, 52: 3406-3410

13 Zhang B, Shi G, Yang Z, et al. Fluorooxoborates: Beryllium-free deep-ultraviolet nonlinear optical materials without layered growth. Angew Chem Int Ed, 2017, 56: 3916-3919

14 Shi G, Wang Y, Zhang F, et al. Finding the next deep-ultraviolet nonlinear optical material: $\mathrm{NH}_{4} \mathrm{~B}_{4} \mathrm{O}_{6} \mathrm{~F}$. J Am Chem Soc, 2017, 139: $10645-10648$

15 Wang $\mathrm{X}$, Wang $\mathrm{Y}$, Zhang $\mathrm{B}$, et al. $\mathrm{CsB}_{4} \mathrm{O}_{6} \mathrm{~F}$ : A congruent-melting deep-ultraviolet nonlinear optical material by combining superior functional units. Angew Chem Int Ed, 2017, 56: 14119-14123

16 Wang Y, Zhang B, Yang Z, et al. Cation-tuned synthesis of fluorooxoborates: towards optimal deep-ultraviolet nonlinear optical materials. Angew Chem Int Ed, 2018, 57: 2150-2154

17 Zhang $\mathrm{X}, \mathrm{Wu} \mathrm{H}, \mathrm{Yu} \mathrm{H}$, et al. $\mathrm{Ba}_{4} \mathrm{M}\left(\mathrm{CO}_{3}\right)_{2}\left(\mathrm{BO}_{3}\right)_{2}$ ( $\mathrm{M}=\mathrm{Ba}$, Sr): two borate-carbonates synthesized by open high temperature solution method. Sci China Mater, 2019, 62: 1023-1032

18 Xie Z, Wang Y, Cheng S, et al. Synthesis, characterization, and theoretical analysis of three new nonlinear optical materials $\mathrm{K}_{7} \mathrm{MRE}_{2} \mathrm{~B}_{15} \mathrm{O}_{30}(\mathrm{M}=\mathrm{Ca}$ and $\mathrm{Ba}, \mathrm{RE}=\mathrm{La}$ and $\mathrm{Bi})$. Sci China Mater, 2019, 62: 1151-1161

19 Huang $C$, Zhang $F$, Cheng $S$, et al. $\alpha-, \beta-\mathrm{Pb}_{4} \mathrm{~B}_{2} \mathrm{O}_{7}$ and $\alpha$-, $\beta-\mathrm{Pb}_{4} \mathrm{~B}_{6} \mathrm{O}_{13}$ : Polymorphism drives changes in structure and performance. Sci China Mater, 2020, 63: 806-815

20 Su J, Zhang J, Tian X, et al. A series of multifunctional coordination polymers based on terpyridine and zinc halide: secondharmonic generation and two-photon absorption properties and intracellular imaging. J Mater Chem B, 2017, 5: 5458-5463

21 Xiong RG, Xue X, Zhao $\mathrm{H}$, et al. Novel, acentric metal-organic coordination polymers from hydrothermal reactions involving in situ ligand synthesis. Angew Chem Int Ed, 2002, 41: 3800-3803

22 Mei J, Leung NLC, Kwok RTK, et al. Aggregation-induced emission: Together we shine, united we soar! Chem Rev, 2015, 115: 11718-11940

23 Jin YJ, Kim H, Kim JJ, et al. Asymmetric restriction of intramolecular rotation in chiral solvents. Cryst Growth Des, 2016, 16: 2804-2809

24 Mei J, Wang J, Qin A, et al. Construction of soft porous crystal with silole derivative: strategy of framework design, multiple structural transformability and mechanofluorochromism. J Mater Chem, 2012, 22: 4290-4298

25 Ma X, Kong W, Abudoureheman M, et al. A homo-chiral helical coordination polymer constructed from an achiral ligand with excellent photo-physical properties and cell imaging application. New J Chem, 2019, 43: 15023-15029

26 Flack HD, Bernardinelli G. Reporting and evaluating absolutestructure and absolute-configuration determinations. J Appl Crystlogr, 2000, 33: 1143-1148

27 Hu Z, Zhang Q, Zhang M, et al. Chiral crystals based on achiral ligand and their framework dependent luminescent properties. Inorg Chem Commun, 2018, 97: 149-156

28 Jin $\mathrm{M}$, Seki $\mathrm{T}$, Ito $\mathrm{H}$. Mechano-responsive luminescence via crystalto-crystal phase transitions between chiral and non-chiral space groups. J Am Chem Soc, 2017, 139: 7452-7455

29 Zhang J, Xue YS, Liang LL, et al. Porous coordination polymers of transition metal sulfides with PtS topology built on a semirigid tetrahedral linker. Inorg Chem, 2010, 49: 7685-7691

30 Dutta B, Maity S, Ghosh S, et al. An acetylenedicarboxylato-bridged $\mathrm{Mn}$ (ii)-based 1D coordination polymer: electrochemical $\mathrm{CO}_{2}$ reduction and magnetic properties. New J Chem, 2019, 43: 5167-5172

31 Xue LP. Synthesis, crystal structure, thermal stability and solid UVVis absorption spectra of one new copper(II) coordination polymer. Chin J Struct Chem, 2019, 38: 1537-1542

32 Zhang JW, Ji WJ, Hu MC, et al. A superstable 3p-block metalorganic framework platform towards prominent $\mathrm{CO}_{2}$ and $\mathrm{C} 1 / \mathrm{C} 2-$ hydrocarbon uptake and separation performance and strong Lewis acid catalysis for $\mathrm{CO}_{2}$ fixation. Inorg Chem Front, 2019, 6: 813-819

33 Zhang LP, Ma JF, Yang J, et al. Series of 2D and 3D coordination polymers based on 1,2,3,4-benzenetetracarboxylate and $\mathrm{N}$-donor 
ligands: synthesis, topological structures, and photoluminescent properties. Inorg Chem, 2010, 49: 1535-1550

34 Chen D, Zhang X, Shi W, et al. Tuning two-dimensional layer to three-dimensional pillar-layered metal-organic frameworks: polycatenation and interpenetration behaviors. Cryst Growth Des, 2014, 14: 6261-6268

35 Zhao X, Wang X, Wang S, et al. Novel metal-organic framework based on cubic and trisoctahedral supermolecular building blocks: topological analysis and photoluminescent property. Cryst Growth Des, 2012, 12: 2736-2739

36 Kurtz SK, Perry TT. A powder technique for the evaluation of nonlinear optical materials. J Appl Phys, 1968, 39: 3798-3813

37 Maggard PA, Nault TS, Stern CL, et al. Alignment of acentric $\mathrm{MoO}_{3} \mathrm{~F}_{3}{ }^{3-}$ anions in a polar material: $\left(\mathrm{Ag}_{3} \mathrm{MoO}_{3} \mathrm{~F}_{3}\right)\left(\mathrm{Ag}_{3} \mathrm{MoO}_{4}\right) \mathrm{Cl}$. J Solid State Chem, 2003, 175: 27-33

38 Brown ID, Altermatt D. Bond-valence parameters obtained from a systematic analysis of the Inorganic Crystal Structure Database. Acta Crystallogr, Sect B: Struct Sci, 1985, 41: 244-247

39 Zhang J, Zhang Z, Zhang W, et al. Polymorphism of $\mathrm{BaTeMo}_{2} \mathrm{O}_{9}$ : A new polar polymorph and the phase transformation. Chem Mater, 2011, 23: 3752-3761

$40 \mathrm{Yu} \mathrm{H}, \mathrm{Wu} \mathrm{H}, \mathrm{Pan} \mathrm{S}$, et al. $\mathrm{Cs}_{3} \mathrm{Zn}_{6} \mathrm{~B}_{9} \mathrm{O}_{21}$ : A chemically benign member of the KBBF family exhibiting the largest second harmonic generation response. J Am Chem Soc, 2014, 136: 1264-1267

Acknowledgements This work was supported by the Natural Science Foundation of Xinjiang Uygur Autonomous Region of China (2019D01C059), the National Natural Science Foundation of China (21671003 and 21201005), the High Performance Computing Center of Henan Normal University and the 111 Project (D17007), Xinjiang Program of Cultivation of Young Innovative Technical Talents (2018Q061), the "2018 Tianchi Doctoral Plan" of Xinjiang Uygur Autonomous Region of China, the Doctoral Scientific Research Foundation of Anhui Jianzhu University (2017QD15) and Xinjiang University. We thank LetPub (www.letpub.com) for its linguistic assistance during the preparation of this manuscript.

Author contributions Zhang J designed the research, performed the synthesis and crystallization, and refined the single-crystal XRD data; Abudoureheman M performed the theoretical data analysis and wrote the manuscript; Ma X and Kong W performed the other experiments; Xuan X and Pan $S$ designed the concept and supervised the experimental and theoretical data collection. All authors contributed to the general discussion.

Conflict of interest The authors declare that they have no conflicts of interest.

Supplementary information Experimental details and supporting data are available in the online version of the paper.

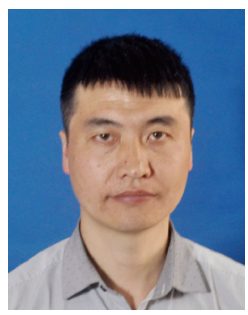

Jun Zhang received his Bachelor's degree from Anhui Normal University, and $\mathrm{PhD}$ degree from Nanjing University. Now he is a full associate professor of Anhui Jianzhu University focusing on the main research of design, construction and functional property for the coordination complexes as well as structural refinement based on single crystal XRD.

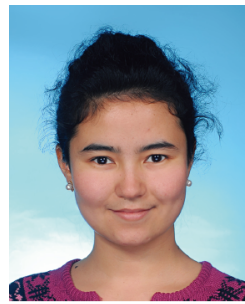

Maierhaba Abudoureheman received her $\mathrm{Ba}$ chelor's degree from Shanghai Jiao Tong University in 2012, and Master's degree from Xinjiang Normal University in 2015. She completed her PhD under the supervision of Professor Shilie Pan at Xinjiang Technical Institute of Physics \& Chemistry (XTIPC), Chinese Academy of Sciences (CAS) in 2018. Now she is a full associate professor of Xinjiang University. She is currently focusing on the optical materials.

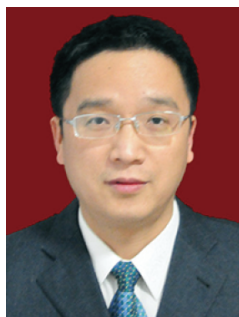

Xiaopeng Xuan received his Bachelor's and Master's degrees from Henan Normal University, and $\mathrm{PhD}$ degree from Lanzhou Institute of Chemical Physics, CAS. Now he is a full professor of Henan Normal University. His research mainly focuses on the thermodynamics of functional solution, and preparation of crystalline materials and their applications.

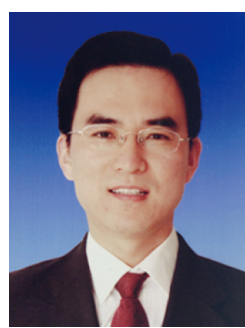

Shilie Pan completed his PhD under the supervision of Professor Yicheng Wu (Academician) at the University of Science \& Technology of China in 2002. From 2002 to 2004, he was a postdoctoral fellow at the Technical Institute of Physics \& Chemistry of CAS in the laboratory of Professor Chuangtian Chen (Academician). From 2004 to 2007, he was a post-doctoral fellow at Northwestern University in the laboratory of Professor Kenneth R. Poeppelmeier in USA. Since 2007, he has been working as a full professor at XTIPC, CAS. His current research interests include the design, synthesis, crystal growth and evaluation of new optical-electronic functional materials.

\section{配位聚合物光学材料的可控合成及自发相变导致} 强二次谐波响应

张俊 ${ }^{1 \dagger^{*}}$, 买尔哈巴·阿不都热合曼 ${ }^{2,4 \dagger}$, 马晓帆 ${ }^{3}$, 孔维 ${ }^{-1}{ }^{1}$, 轩小朋 $^{3^{*}}$, 潘世烈 ${ }^{* *}$

摘要 本文以具有聚集诱导发光(AIEgen)的三联吡啶衍生物为配 体, 构建了一种新型配位聚合物 (CP)光学材料. 该材料可以从中心 对称结构(1- $\boldsymbol{\alpha}$ 相) 自发相变成非中心对称结构(1- $\boldsymbol{\beta}$ 相), 两种物相均 能可控合成, 且通过单晶X射线衍射清晰表征相变前后结构. 有趣 的是, 中心对称 $\mathbf{1}-\boldsymbol{\alpha}$ 相转变为非中心对称 $\mathbf{1 - \beta}$ 相, 从而产生强相位匹 配二次谐波 $(\mathrm{SHG})$ 响应, 约为 $\mathrm{KH}_{2} \mathrm{PO}_{4}(\mathrm{KDP})$ 的 4.5 倍. 为了更好地理 解结构与非线性光学特性之间的关系, 对偶极矩进行了计算和讨 论. 值得注意的是, 1- $\boldsymbol{\beta}$ 具有高热稳定性的非中心对称结构, 在 $\mathbf{1 - \alpha}$ 结构相变后保留并改善了AIEgen配体的初始发光性质, 同时产生 了新的优异的SHG性质, 这为设计和制备优良的光学材料提供了 一条很好的途径. 